\title{
A Preliminary Nonlinear Analysis of the Earth's Chandler Wobble
}

\author{
V. FREDE ${ }^{a, *}$ and P. MAZZEGA ${ }^{b}$ \\ ${ }^{a} U M R 8630$ Observatoire de Paris, 61, avenue de l'Observatoire, 75014 Paris, France; \\ ${ }^{\mathrm{b}} U M R$ 5566-CNRS-CNES, 18 av. E. Belin, 31401 Toulouse cedex 4, France
}

(Received 31 January 1999)

\begin{abstract}
The Chandler wobble $(\mathrm{CW})$ is a resonant response of the Earth rotational pole wandering around its figure axis whose excitation mechanism is still uncertain. It appears as polar motion oscillations with an average period of about 433 days and a slowly varying amplitude in the range ( $0-300)$ milliarcsec (mas). We here perform a nonlinear analysis of the $C W$ via a time-delay coordinate embedding of its measured $X$ and $Y$ components and show that the $C W$ can be interpreted as a low dimensional unstable deterministic process.

In a first step the trend, annual wobble and $\mathrm{CW}$ are separated from the raw polar motion data time series spanning the period 1846-1997. The optimal delays as deduced from the average mutual information function are 105 and 115 days for the $X$ and $Y$ components respectively. Then from the global statistics of the false neighbours, the embedding dimension $D_{\mathrm{E}}=4$ is estimated for both series. The local dimension $D_{\mathrm{L}}$ can also be extracted from the time series bv testing the predictive skill of local mappings fitted to the embedded data vectors. The result $D_{\mathrm{L}}=3$ is quite robust and corroborate the idea that the $\mathrm{CW}$ behaves like a dissipative oscillator driven by a deterministic process. Indeed the orbit reconstructions in pseudo-phase space both draw the figure of a perturbated 1-torus.

The computation of the Lyapunov spectra further shows that this torus-like figure is an attractor with a 1D unstable manifold. The theoretical horizons of prediction deduced from the (positive) principal exponents are about 367 and 276 days for the $X$ and $Y$ Chandler components respectively. Moreover the local Lyapunov exponents exhibit significant variations with maxima (and corresponding losses of predictibility) in the decades 18601870 and $1940-1950$.
\end{abstract}

Keywords: Chandler wobble, Chaos, Lyapunov exponents, Time series

\section{INTRODUCTION}

The Earth rotation rate and spin axis position relative to the Earth's figure axis, experience fluctuations at all time scales. Variations in the length of day (LOD) and polar motion (PM) are observed in response to applied external (e.g. tidal) and internal (e.g. topographic coupling between

\footnotetext{
*Corresponding author. E-mail: fred@hpvlbi.obspm.fr.
} 
core and mantle) torques, to angular momentum exchanges between the solid and fluid parts of the Earth and to global mass redistributions primarily associated to geophysical fluid motions which perturb the Earth inertia tensor (Lambeck, 1980). Nowadays, the high accuracy geodetic measurements of these fluctuations provide useful constraints on the time evolution of the Earth-Moon system and on the modelling of the earth's core and mantle as well as hydrosphere and atmosphere dynamics (Hide and Dickey, 1991; Dehant et al., 1997).

The Chandler wobble $(\mathrm{CW})$ is a resonant response of the PM. The corresponding free oscillation was already predicted by Euler in 1765 with a period of about 306 solar days for an axisymmetric flattened rigid Earth model. But it was only observed by Chandler in 1891 from data of the variation of latitudes, with a period of about 14 months ( $\sim 436$ days). This increase of the period is well accounted for by the elastic yielding of the Earth. Several processes in the Earth's global dynamics being dissipative (e.g. the wind and oceanic current drag on the crust), the $\mathrm{CW}$ must be sustained by an excitation source which has resisted any reliable identification until today.

Several candidates have been proposed in the past but none of them appear to be appropriate. The energy input by earthquakes is probably between two or five orders of magnitude too small to excite the wobble (Souriau, 1986; Chao and Gross, 1987). Other possible excitation mechanisms were searched for in pre- or post-seismic deformations (Sabadini et al., 1984) and in convective processes of the core external layers (Le Mouel et al., 1985) without reaching conclusive results. Early investigations about the possible excitation by atmospheric or oceanic variabilities (Ooe, 1978; Wahr, 1983; Chao, 1984) are somewhat supported by recent advances in global ocean-atmosphere modelling (Vondràk, 1990; Chao, 1993) but with rather mitigated results. Significant departures between the predicted and observed wobble are still unexplained so that other mechanisms might at least partly - sustain the Chandler oscillation (Eubanks, 1993).
Other studies have been dedicated to the characterization of the $\mathrm{CW}$ from the data series. Variations in amplitude and departures from a purely periodic motion are reported since a long time (see e.g. Jeffreys, 1940). Frequency modulations were also sought with diverging conclusions that should result from differences in the way data were analyzed and from data contaminations by a time-dependent noise. Indeed phase variations of the CW could be an artifact of observational errors or result from random unknown excitations (Okubo, 1982). Two or more free frequencies could also coexist near the Chandler frequency supposed constant and modulate the signal (Colombo and Shapiro, 1968). A single Chandler frequency might be amplitude-dependent due to nonlinear response to the oceans and change with time (Carter, 1981).

Major information on the CW energy sources and sinks can be gained from the estimation of its quality factor $Q_{\mathrm{CW}}$. This factor, which is inversely proportional to the ratio of the energy loss $-\Delta E_{\mathrm{CW}}$ in one wobble cycle to the energy $E_{\mathrm{CW}}$ of an unsustained wobble, can be retrieved from the width of the CW spectral line (Stacey, 1992). Recent estimates of $Q_{\mathrm{CW}}$ range from $\sim 50$ to $\sim 180$ (Furuya and Chao, 1996) that correspond to decays of an unmaintained CW with typical time scales of $\sim 20$ 70 years respectively.

Until now almost all analyses aiming at the identification of excitation sources or at extracting $\mathrm{CW}$ parameters from the data were conceptually confined to the "stochastic process paradigm". However the resonant response of a damped oscillator is stronger when the forcing has a rich spectral content about its free period. Following this idea, quasi-periodic angular momentum fluctuations in the atmosphere near the 14 month period were recently retrieved by Furuya et al. (1996). Unfortunately their study relies on linear analyses (Fourier spectra, coherence functions) though it is known that the dynamics of the various geophysical fluids (core, mantle, ocean, groundwater, atmosphere) are driven by nonlinear processes at sub-daily to climate time scales. 
Results presented here suggest that the $\mathrm{CW}$ is sustained by a low dimensional nonlinear deterministic process. The nonlinear data time series analysis of the rapid fluctuations of the LOD and PM in the frequency band $(2-100)$ days already supported a similar conclusion (Frede and Mazzega, 1999a). In particular nonlinear signatures of El Niño Southern Oscillation events (Harrison and Larkin, 1998) were recovered in the time-dependent local Lyapunov exponents, indicating that at these periods the unstable modes of the Earth's rotation are prompted by the ocean-atmosphere coupled dynamics (Frede and Mazzega, 1999b).

In the next section we present the data time series and explain how the $\mathrm{CW}$ signal is routinely separated from the annual wobble, trend and residues. In Section 3, the embedding $D_{\mathrm{E}}$ and local $D_{\mathrm{L}}$ dimensions are determined from global and local neighbourhood analyses of the embedded data vectors. The Lyapunov exponents and dimensions are then computed from the data time series as explained Section 4 and the results discussed in Section 5. However this analysis should be considered as preliminary. Indeed we do not tried here to test the robustness of these results. Similar computations should also be undertaken with PM data time series published by other Earth's rotation center. The algorithms and sensitivity tests (to noise contaminations, limited data series, etc.) are detailed in the context of Earth's rotation studies in the twin papers by Frede and Mazzega (1999a,b). From our experience, we think that the results presented below are robust and ascertain the nonlinear determinist origin of the $\mathrm{CW}$ excitation mechanism.

\section{TIME SERIES OF THE CHANDLER WOBBLE (1846-1997)}

The CW is extracted from data series of the raw PM components $X$ (along the Greenwich meridian) and $Y$ (along the $90^{\circ} \mathrm{E}$ meridian) as edited by the International Earth Rotation Service (IERS, Observatory of Paris). The longest available series (the C01 series, see IERS, 1997) extends from 1846 up to 1997. During this one and half century of survey the techniques of PM observation have considerably evolved and the resulting C01 series is a concatenation or, when possible, an optimal combination of partial data series (Vicente and Wilson, 1997). For example in the early period (1846-1890) the PM is deduced from astrometric optical measurements of absolute declinations with a frequency band covering almost only the Chandler and annual wobbles and an accuracy probably not better than a few tens of milliarcsec (mas). The latest two decades of data combine geodetic observations from Very Long Base Interferometry (VLBI), Global Positioning System (GPS) and Satellite and Lunar Laser Ranging (SLR and LLR respectively, IERS, 1997) with an accuracy of 0.3 mas. The sampling rate is $10 \mathrm{data} /$ year from 1846 to 1889 and 20 data/year from 1890 up to now.

The PM is dominated by two main components: the annual wobble which is presumably excited by the ocean-atmosphere system and the CW. They can be quite well separated from each other with a 7 year-long record of $\mathrm{PM}$ as during such a time interval 6 full cycles of the $\mathrm{CW}$ are completed. The splitting of a time series into trend, seasonal and irregular components is a practical issue in financial time series analysis. The Census X-11 filter (Shiskin et al., 1967) achieves this kind of decomposition on the basis of local trend estimators for non-seasonal time series using finite moving averages (Gray and Thomson, 1997). It is routinely implemented in an iterative data analysis scheme at IERS in order to separate the annual and Chandler wobbles (see IERS, 1991, for details).

In a first step, the $X$ and $Y$ time series of the PM have been re-interpolated with a homogeneous sampling rate of 100 data/year all over the (18461997) interval with a cubic spline. Then we applied the iterative decomposition with the finite moving averages being estimated over moving 7-year subintervals in order to separate the annual and Chandler wobble without smothing too much the time variability of these signals. We retain a central 
period of 433 solar days for the $\mathrm{CW}$ in good agreement with the recent determinations of this parameter (see e.g. Vicente and Wilson, 1997). It should be noted that the frequency resolution of the moving averages over 7-year time span is quite low so that weak frequency modulations of the $\mathrm{CW}$ about its central frequency are implicitly permitted in the decomposition process. The annual wobble is about 60 mas RMS, the CW about 100 mas RMS and the irregular part about 40 mas RMS on both components of the PM (means and RMS are summarized in Table I). The statistics of the wobbles and irregular parts of the $X$ and $Y$ series are similar. The main difference between the two raw series is a consequence of the strong trend in the $Y$ component of the PM $(\sim 4$ mas/year since the beginning of this century). We will not be interested in this secular drift that might be caused by the postglacial rebound or changes in polar ice covering (McCarthy and Luzum, 1996).

The raw series, trend, wobbles and irregular part are drawn in Fig. 1 for the $Y$ component (similar curves are obtained with the $X$ component of $P M$ ). The quite high noise level in the years (1846-1890) is responsible for 40 mas RMS of the irregular (or residual) part of PM. It would rather be of the order of 10 mas RMS when considering the data obtained after 1890 . The noise is also spoiling the annual and CW recovery before 1890 as can be seen on Fig. 1. Then the most striking phenomenon is the low amplitude of the CW in the years (1925-1940). This strong modulation has been interpreted as a phase reversal of the wobble (see e.g. Vondràk, 1990) whose origin is still unknown (this modulation occurs on the $X$ component as well). In fact the

TABLE I Mean and RMS of the raw series of the $X-Y$ components of the PM and of their respective trend, CW, annual wobble and irregular part (unit: mas)

\begin{tabular}{lcc}
\hline PM decomposition & Mean $X-Y$ (mas) & RMS $X-Y$ (mas) \\
\hline Raw series & $-15.7 \| 49.8$ & $142.9 \| 188.4$ \\
Trend & $-15.3 \| 50.3$ & $39.7 \| 135.0$ \\
Chandler & $0.0 \| 0.0$ & $102.9 \| 101.4$ \\
Annual & $0.0 \| 0.0$ & $65.0 \| 57.7$ \\
Irregular & $-0.3 \|-0.5$ & $41.3 \| 42.4$ \\
\hline
\end{tabular}

time variations of the excitation mechanism should be searched in these slow modulations of the resonant response of the Earth's rotation (and may be on related frequency modulations that are more difficult to track).

A detailed examination of the annual and Chandler wobbles also reveals that these two components might not be perfectly separated by the Census X-11 algorithm. This is not so surprising as we operate the decomposition over the smallest possible moving window of 7 years. In the computations described below, the first 10 years of data are skipped in order to limit the effect of the data noise on the results. Moreover a weak smoothing via local parabolae fitting to data included in a (moving) window of 150 days, is applied to the Chandler series before entering the nonlinear analysis. The instantaneous wobble is taken as the value of the fitted local parabola middle point. This filtering only reduces the $X$ and $Y \mathrm{CW}$ series by $\sim 2$ mas RMS though removing most of the residual noise contaminations in the data series.

\section{DIMENSIONAL ANALYSIS OF THE CHANDLER WOBBLE}

Dimensional and dynamical invariants of a complex system can be extracted from the nonlinear analysis of an univariate data time series issued from that system. Data vectors are constructed in a

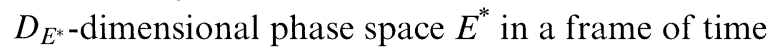
delayed coordinates. In $E^{*}$ the time series of data vectors draws an orbit whose statistical and geometrical properties, thanks to the embedding theorem (Takens, 1981), we know to be directly inherited from the unknown source dynamics (Eckmann and Ruelle, 1985). The deep theoretical background of this approach will not be addressed at all in this study. The interested reader will refer to the papers cited above or to the recent overview given by Abarbanel (1996).

Here the complex system is the Earth with its various interacting envelopes (solid parts, geophysical fluids, etc.) that we study from the point of view 
Split Y-C01 (units: mas)
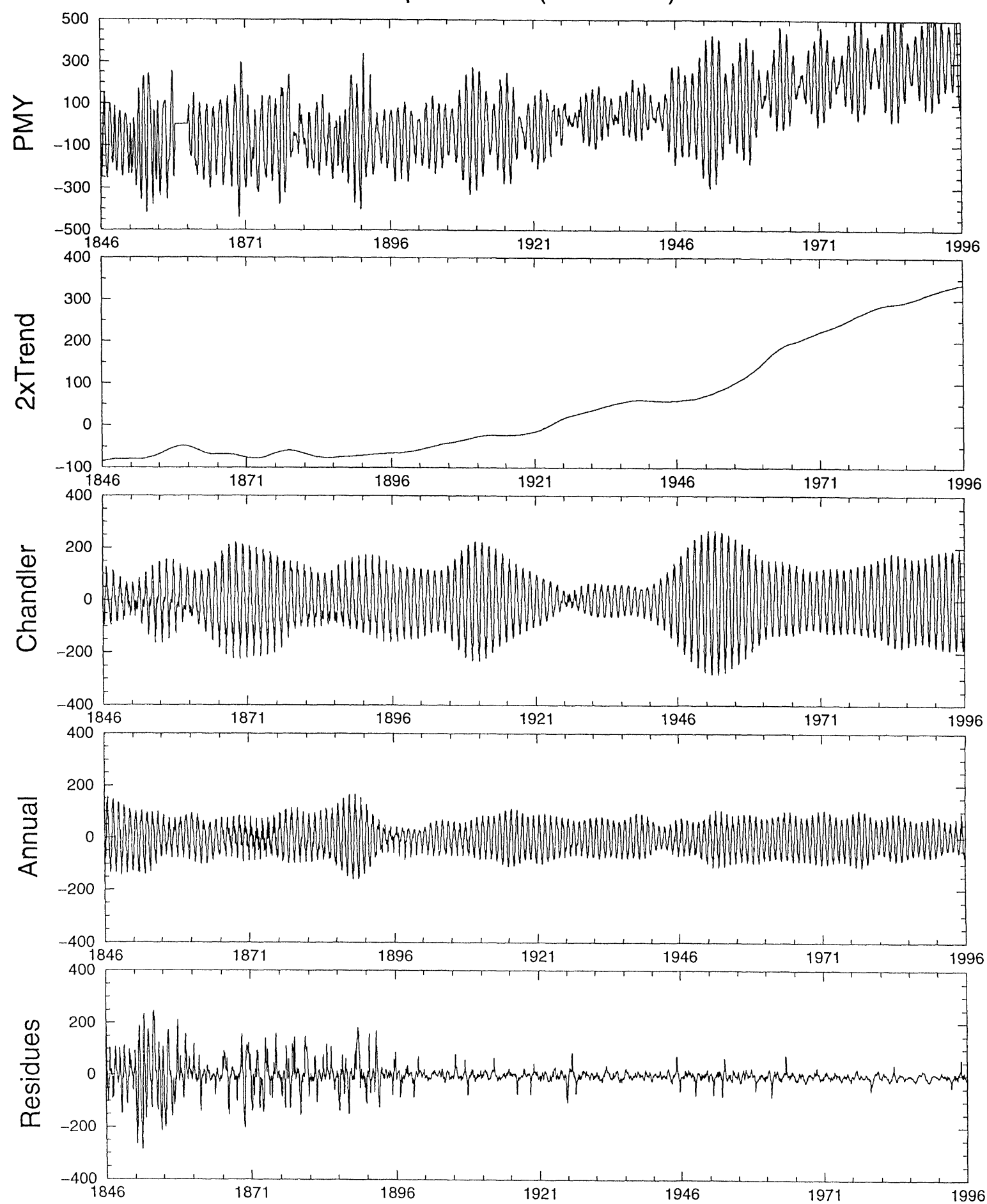

FIGURE 1 The raw time series of the Y-PM (upper panel) is splitted into a secular trend (scaled by a factor 2), the Chandler (period $\sim 433$ days) and annual (period 365.25 days) wobbles and an irregular or residual part (lower panel). Units are in mas. Very similar plots are obtained with the $X$ component of PM. 
of its rotational dynamics. In the computations below, we consider separately the $X$ and $Y$ components of the $\mathrm{CW}$. Being extracted from the same dynamics they should lead us to the same estimates of the system invariants. The series of the $\mathrm{CW}$ being limited in length and contaminated by noise, the coherence of the results extracted from both series is a crude way to give some confidence in the characterizations so obtained.

The dimensional analysis starts with the determination of the smallest dimension of the space $E^{*}$ required to unfold the reconstructed orbits. Note that this dimension $D_{E^{*}}$ might depend on the data series extracted from the system and so is not an invariant of the dynamics. From the data time series at hand $x(t)$ we construct $D_{E^{*}}^{\text {trial }}$-dimensional vectors $y(t)$ using a time delay $\tau$ by:

$$
y(t)=\left[x(t), x(t+\tau), \ldots, x\left(t+\left(D_{E^{*}}^{\text {trial }}-1\right) \tau\right)\right] .
$$

In $E_{\text {trial }}^{*}$, the time series of the vectors $y(t)$ draws some parametric curve with a lot of neighbour points (or even intersections). These neighbourhoods, which can be easily counted, result either from the system passing again similar states in phase space during its evolution or from undesirable projections of the reconstructed orbit in a too low dimensional space. These "false" neighbours are progressively eliminated when increasing the trial embedding dimension $D_{E^{*}}^{\text {trial }}$. Only true neighbours remain as soon as the smallest dimension required to completely unfold the orbit, say $D_{E^{*}}$, is reached. Stochastic processes being infinite dimensional, their data contamination tends to give an overestimated embedding dimension, and even sometimes compromises its determination (Frede and Mazzega, 1999a).

In Eq. (1), the definition of a system of statistically independent coordinates relies on the choice of the time-delay $\tau$. In the context of nonlinear dynamics, it has been shown that the best way to estimate a reliable delay is to calculate the Average Mutual Information (AMI) function (Fraser and Swinney, 1986) which, shortly speak- ing, is based on the probability distribution of events in the time series. The joint probability distribution between the data series $x(n)$ and its delayed version $x(n+T)$, say $P[x(n), x(n+T)]$ is obtained from the joint histogram in the $[x(n)$, $x(n+t)$ ] variables (for simplicity we here assume that the data are given at discrete times $n=1, N$ ). The AMI for any delay $T$ is defined by:

$$
\begin{aligned}
& \operatorname{AMI}(T) \\
& \quad=\sum_{n=1}^{N} P[x(n), x(n+T)] \ln \left[\frac{P[x(n), x(n+T)]}{P[x(n)] P[x(n+T)]}\right] .
\end{aligned}
$$

The first minimum of the AMI function is the sought delay. We obtain time delays of 105 and 115 days respectily for the $X$ and $Y$ components of the $\mathrm{CW}$. It can be seen from the AMI function for $Y$ plotted in Fig. 2, that the minimum is surrounded by weak gradients. So the estimation of $\tau$ is not very acute and could easily vary within \pm 10 days or so. Anyway, the various orbits that would be reconstructed on the basis of slightly different time delays are diffeomorphic and equally lead to the sought invariant estimates.

With the optimal delay for a given series, we build data vectors as in Eq. (1) for a trial dimension $D_{E^{*}}^{\text {trial }}$. For each vector, we count the number of neighbour vectors and compare it to the number of neighbours

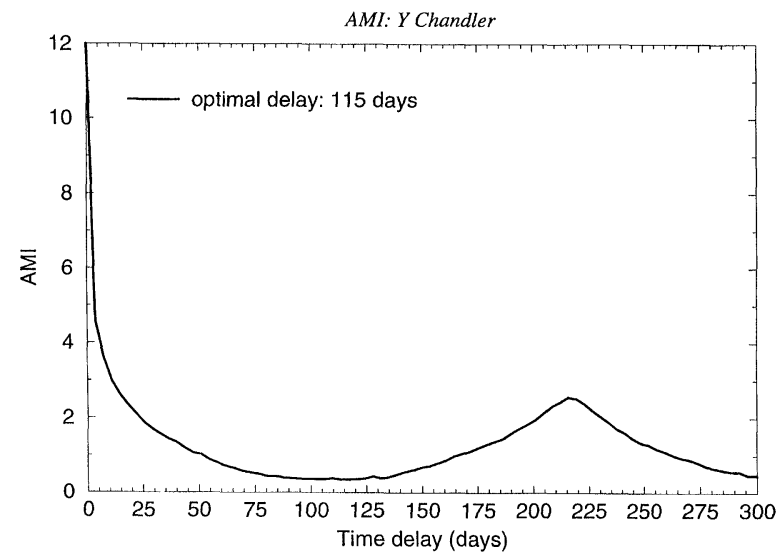

FIGURE 2 AMI as a function of the time-delay $T$. The delay $\tau$ for data embedding corresponds to the first minimum of the AMI function. 


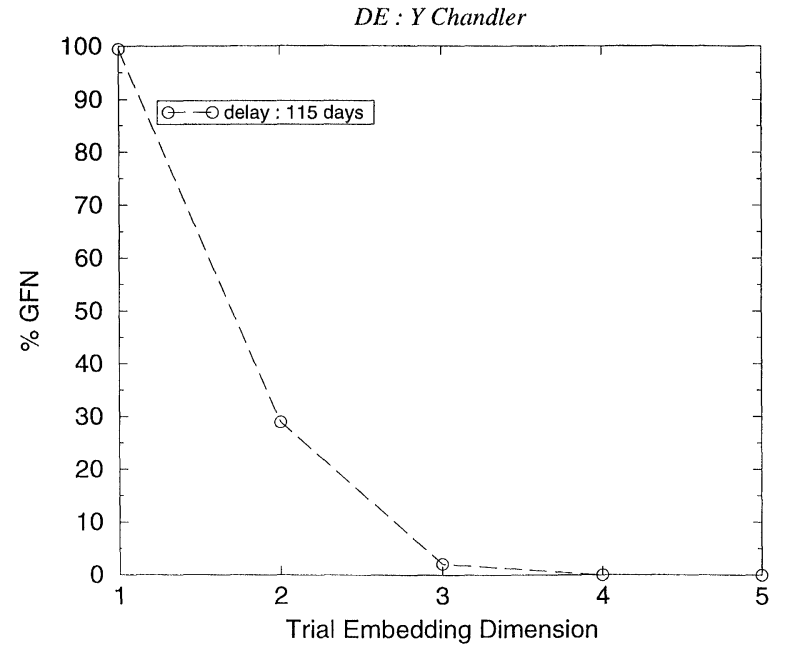

FIGURE 3 Global percentage of false neighbours as a function of the trial embedding dimension $D_{E^{*}}^{\text {trial }}$ for the $Y$-Chandler data time series. The $0 \%$ in dimension $D_{E^{*}}=4$ corresponds to the complete unfolding of the reconstructed orbit in pseudo-phase space $E^{*}$.

in dimension $D_{E^{*}}^{\text {trial }}+1$. Those neighbourhood of dynamical origin are not affected while those resulting from a projection are gradually eliminated by the unfolding of the embedding space. In Fig. 3 we plot the global percentage of false neighbours as a function of the trial embedding dimension $D_{E^{*}}^{\text {trial }}$ for the $Y$ series with a delay $\tau=115$ days (once again similar plots are systematically obtained for the $X$ series). Almost all the neighbours in dimension 1 are false neighbours (globally $\sim 100 \%$ of false neighbours) as the data are artificially projected on a line. This percentage decreases to zero for a trial dimension of 4 . At this stage it is difficult to know if the very low percentage in dimension 3 is not a remanent of data contamination by a weak noise. However, exactly the same behaviour being observed for the $X$ component, we retain an embedding dimension $D_{E^{*}}=4$ in the following. It can be shown that these results are not sensitive to the value of the radius used to define any neighbourhood as long as it is chosen as a small fraction of the data series RMS.

From the knowledge of the time-delay $\tau$ and embedding dimension $D_{E^{*}}$, the orbit is reconstructed in pseudo-phase space $E^{*}$. A $3 \mathrm{D}$ projection of the $Y$-Chandler orbit is drawn in Fig. 4. It looks like interweaving "circles" more or less confined in a bounded disk-like sub-space. Much will be learnt about the dynamics of the CW from the geometry of such a reconstructed orbit.

The next dimension to be determined is the local dimension. Whereas the embedding dimension $D_{E^{*}}$ is a global dimension, the dynamical dimension $D_{\mathrm{L}}$ is related to the exact number of degrees of freedom activated during the system evolution. It is an invariant of the system dynamics that can be also extracted from a time series of observations. The broad lines of the algorithm are summarized as follows (see Abarbanel and Kennel, 1993 for details): the orbit is reconstructed in a working space with high enough dimension $D_{\mathrm{w}}>D_{E^{*}}$ to ascertain that true neighbours only are kept. We then consider a given point of the orbit, say $y(n)$, select its $N b$ nearest neighbours $\left[y_{i}^{\mathrm{nghb}}(n) ; i=1, N b\right]$ and compute the corresponding covariance matrix. The eigenvectors of this matrix give the principal local directions along which are distributed the selected data vectors. Keeping only the $D_{\mathrm{L}}^{\text {trial }}$ local directions, we build a maping which carries the vectors $y_{i}^{\text {nghb }}(n)$ to their images $y_{i}^{\text {nghb }}(n+m)$ after $m$ time steps of the ambient flow. This mapping is finally applied to the vector $y(n)$ and the result $y_{\text {pred }}(n+m)$ is compared to its true image $y(n+m)$. The prediction is considered successful if the euclidian distance $\left\|y(n+m)-y^{\text {pred }}(n+m)\right\|$ is below a predefined fraction of the data series RMS (typically $10 \%$ ). These operations are repeated for all the points $y(n)$ of the orbit and the percentage of bad predictions is estimated. All the predictions are successful only when the local mappings are built in the right dynamical dimension $D_{\mathrm{L}}$. In this case the performance of the prediction does not depend on the number of neighbours $N b$ nor on the success criterion.

The choice of $\mathrm{Nb}$ for a limited time series in the presence of noise contamination is discussed in Frede and Mazzega (1999b). Indeed we found this algorithm to be quite sensitive to noise because it basically works with neighbourhoods so that the local evolution of the flow is partially hidden by noisy features of the reconstructed orbit. The 


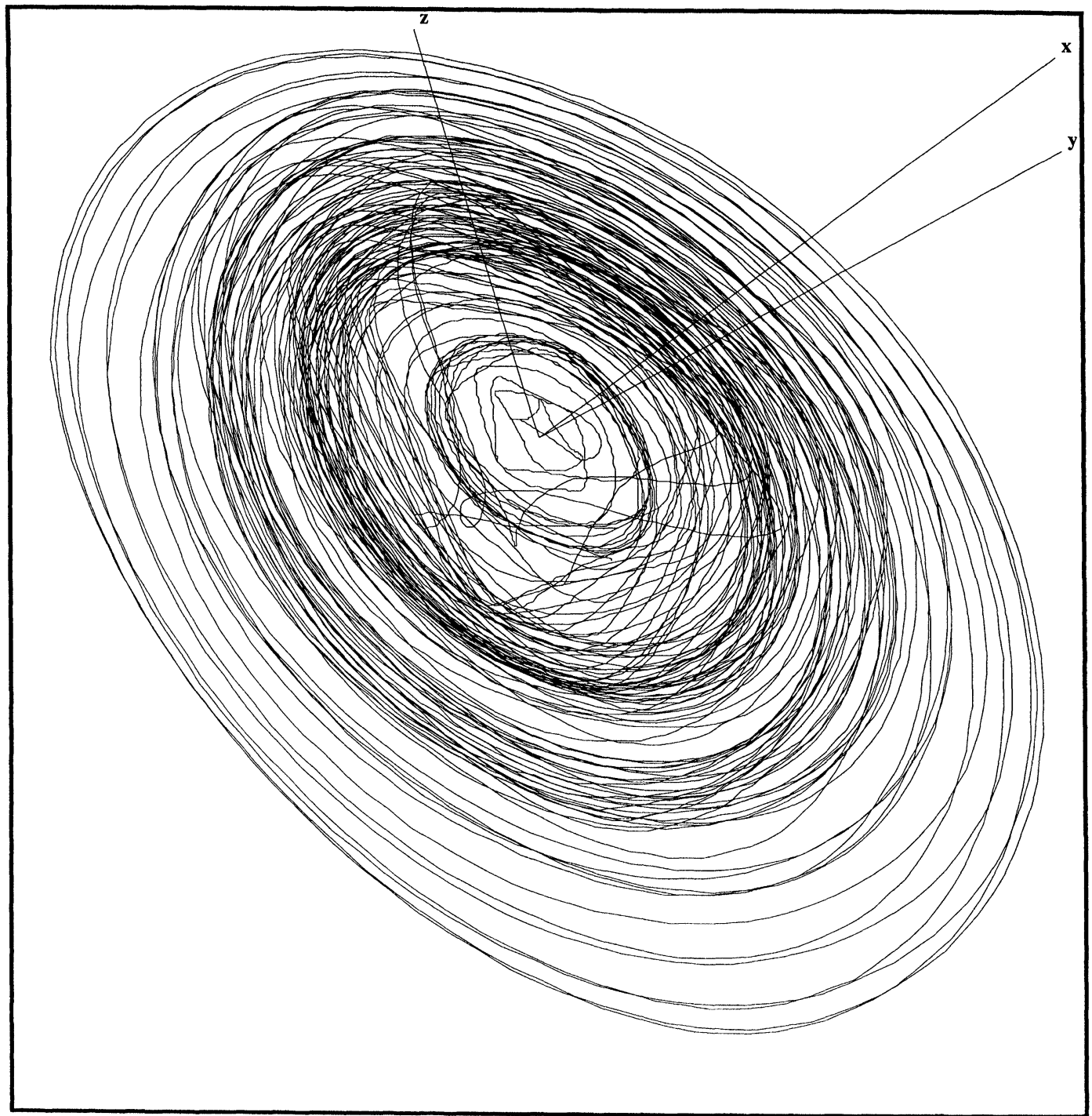

FIGURE $4 \quad Y$ component of the CW reconstructed as an orbit in 4D pseudo-phase space $E^{*}$ (one of its 3D projection is drawn here). The delay for embedding is 115 days. The radius of the torus-like structure is about twice the data series RMS ( 100 mas).

algorithm applied to the complete Chandler series spanning the period (1847-1997) gives no results: the percentage of bad predictions remains about $25-40 \%$ whatever the trial local dimension is. It converges to $D_{\mathrm{L}}=3$ when skipping the first 10 years of observations of the $Y$ component (Fig. 5). Thirty years of data (1847-1876) have to be skipped for observing a convergence to the $0 \%$ level of bad prediction for the $X$ component of the $\mathrm{CW}$. Once again the corresponding local dimension is $D_{\mathrm{L}}=3$. A few tens of years of very noisy observations (see the residues in Fig. 1) can spoil the whole determination of $D_{\mathrm{L}}$ because such a data sub-series is whirling several times around the attractor draw in Fig. 4 and contaminates the decomposition of all the local data covariance matrices. Indeed in phase space $E^{*}$ the set of the nearest neighbours to some recent accurate data vector often includes old and 


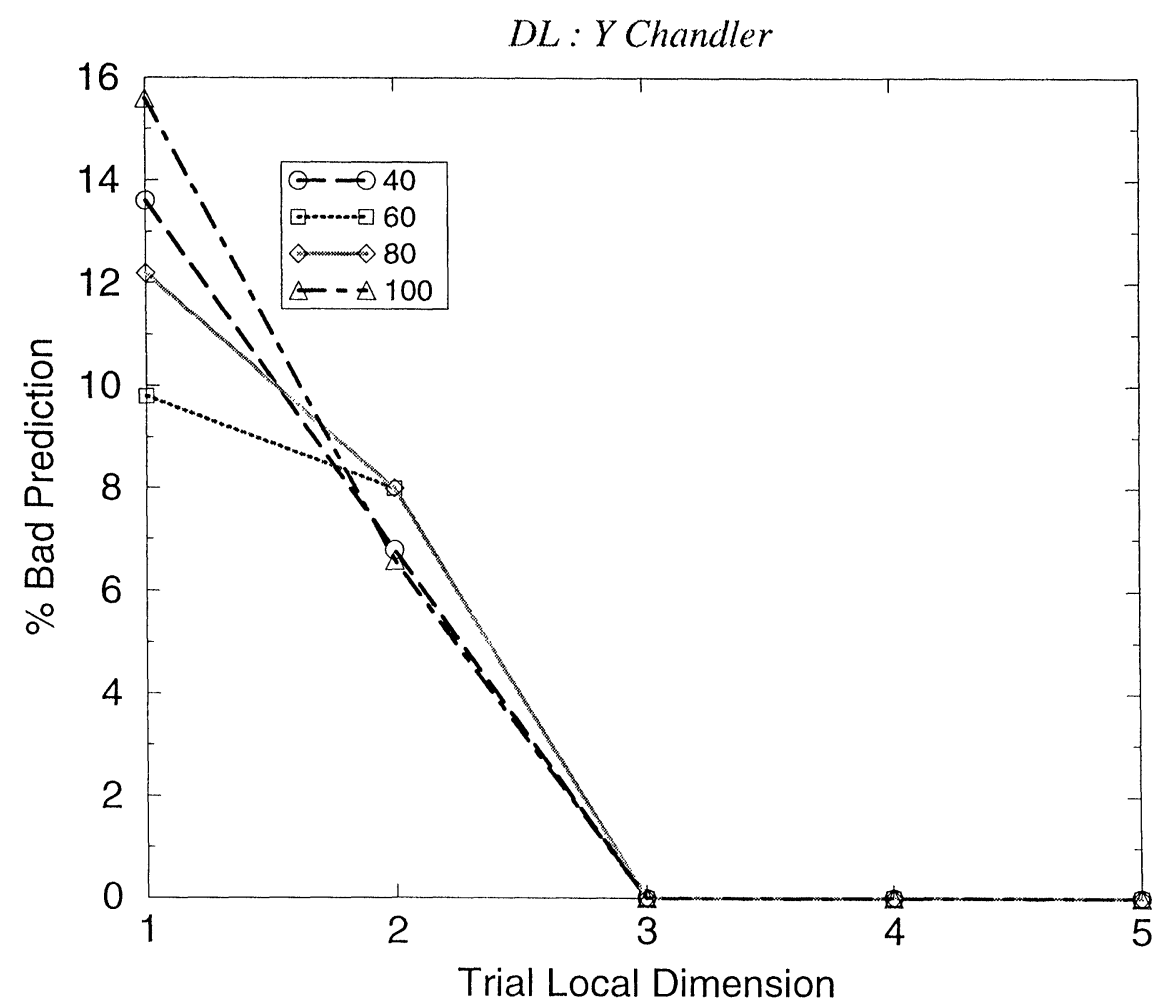

FIGURE 5 Percentage of bad predictions as a function of the trial local dimension $D_{\mathrm{L}}^{\text {trial }}$ for the $Y$-Chandler data time series. The $0 \%$ prediction error level reached in dimension $D_{\mathrm{L}}=3$ does not depend on the number of nearest neighbours fixed to build the local covariance matrices (see text) and indicated in the window.

noisy measurements. Anyway, the removal of the too noisy early data ensures the convergence of the local dimension determination to the value $D_{\mathrm{L}}=3$ for both series.

\section{STABILITY ANALYSIS OF THE CHANDLER WOBBLE}

Information related to the stability of the observed $\mathrm{CW}$ can also be gained from the nonlinear data analysis. The first point is to understand what can be the fate of a small perturbation of the wobble orbit, say $\delta y$ : will it fade away, remain unchanged or grow with time? These three possibilities, encapsulated in the following formula:

$$
\delta y(t)=\delta y(0) \exp [\lambda t]
$$

are clearly determined by the sign of the Lyapunov exponent $\lambda$ (here $\delta y(0)$ is the perturbation of the reconstructed orbit at an arbitrary time considered as "initial" and $\delta y(t)$ the perturbation at time $t$ ). Any infinitesimal perturbation taken along the direction locally tangent to the orbit, cannot grow nor fade with time as the perturbated state $y+\delta y$ belongs to the same orbit and so actually corresponds to a near past or near future state of the system. As a consequence the Lyapunov exponent associated to this neutral direction is zero, a value that should be retrieved from the data (up to noise contamination). The $\mathrm{CW}$ being modellized by a three degrees of freedom system $\left(D_{\mathrm{L}}=3\right)$, two more directions must be considered that can be associated to stable (negative exponent) or unstable (positive exponent) manifolds. The wobble resulting from the interaction of dissipative processes, the sum of the 
exponents must he negative, a condition that expresses the overall contraction of volumes in phase space and the confinement of orbits in a bounded domain.

Without entering technical details, we briefly report how the Lyapunov spectrum can be extracted from a data series. Consider the $\mathrm{CW}$ as a dynamical system observed at discrete times indexed by integers $i$ :

$$
y_{i+1}=F\left(y_{i}\right) \text {. }
$$

After $K$ time steps, the perturbation $\delta_{i}$ applied to $y_{i}$ is transformed by the composed action of the local jacobian matrices $D F$ of the dynamics along the orbit, say:

$$
\begin{aligned}
\delta_{i+K} & =D F\left(y_{i+K-1}\right) \cdot D F\left(y_{i+K-2}\right) \cdots D F\left(y_{i}\right) \cdot \delta_{i} \\
& \equiv D F^{K}\left(y_{i}\right) \cdot \delta_{i} .
\end{aligned}
$$

It can be shown that the sought Lyapunov exponents are related to the eigenvalues of the Osedelec matrix $S\left(y_{i}, K\right)$ (see e.g. Eckmann and Ruelle, 1985) defined by:

$$
S\left(y_{i}, K\right)=\left[D F^{K}\left(y_{i}\right)^{\mathrm{T}} \cdot D F^{K}\left(y_{i}\right)\right]^{1 /(2 K)},
$$

where the superscript $\mathrm{T}$ is for transpose. Besides those computational difficulties involved in the decompostion of $S$ because of its intrinsic bad conditioning (see Abarbanel, 1996 for algorithmic details), the important point to be noted here is that the $D F$ 's can be estimated from the data time series, without prior knowledge of the dynamics in closed formulae. They are obtained from the partial derivatives of local maps

$$
y_{i}^{v} \rightarrow y_{v ; i+1}
$$

developed on basis functions so to fit the time evolution of the embedded data vectors $\left(y_{v, i+1}\right.$ is the $v$ th neighbour of $y_{i}$ at time $\left.i+1\right)$. The global exponents are obtained from the Oseledec matrix which spans all the data series (starting from $y_{1}$ and
$K$ being the length of the vector series). Theoretically the Lyapunov spectrum is invariant with regard to changes in the initial conditions, as long as the data series is infinite $(K \rightarrow \infty)$. The possible effects of series finiteness and noise contaminations on the estimation and reliability of Lyapunov exponents are empirically studied and discussed in a similar context in Frede and Mazzega (1999b). We here just mention that our series seem to be long enough and not too much spoiled by noises so that significative estimates of the three exponents can be extracted. The coverage of the phase space subvolumes visited by the $\mathrm{CW}$ system is dense enough and allows the proper computations to be robust. This statement will be further documented below when considering time-reversed data series. However, the genuine "global" Lyapunov exponents of the $\mathrm{CW}$ are definitely beyond the observational grasp. The exponents we actually compute are average values over the measurement time span.

The Lyapunov exponents for the $X$ and $Y$ components of the CW have been computed. For both series the principal exponent $\lambda_{1}$ is positive $\left(\lambda_{1}^{+}(X)=0.046\right.$ and $\lambda_{1}^{+}(Y)=0.086$ respectively, see Table II). The exponent associated with the neutral manifold is smaller than 0.01 in absolute value for $X$ and $Y$. These departures from the exact zero value result from the series finiteness, noise contaminations and numerical approximations.

TABLE II Numerical characteristics of the nonlinear dimensional and stability analysis of the $X$ and $Y$ components of the $\mathrm{CW}$

\begin{tabular}{lcc}
\hline Characteristic & $X$-Chandler & $Y$-Chandler \\
\hline$\tau$ (days) & 105 & 115 \\
$D_{E^{*}}$ & 4 & 4 \\
$D_{\mathrm{L}}$ & 3 & 3 \\
$D_{\mathrm{u}}$ & 1 & 1 \\
$\lambda_{\mathrm{I}}+/-$ & $0.046 \| 0.032$ & $0.086 \| 0.018$ \\
$\sum \lambda_{\mathrm{i}}+/-$ & $-0.073 \|-0.026$ & $-0.103 \|-0.024$ \\
$D_{\text {Lyap }}$ & 2.43 & 2.42 \\
$H_{\mathrm{P}}$ (days) & 386 & 276 \\
\hline
\end{tabular}

Time-delay $\tau$ deduced from the AMI function; embedding $D_{E^{*}}$ and local $D_{\mathrm{L}}$ dimensions; dimension of the unstable manifold $D_{\mathrm{u}}$; principal Lyapunov exponent $\lambda_{1}+/-$; sum of the Lyapunov exponents $\sum \lambda_{i}+1-;$ Lyapunov dimension $D_{\text {Lyap }}$ and horizon of the prediction $H_{\mathrm{P}}$. The ' + ' and '-' refer to the forward and backward time series respectively. 
However it is well below those estimates of the $\lambda_{1}$ exponents which are, from a computational point of view, better approximated. As expected, both third exponents are associated with stable manifolds ( -0.115 for $X$ and -0.179 for $Y$ ), the wobble process being dissipative and the sum of the exponents being negative (Table II).

Apart from the exact value of $\lambda_{1}$, the key information is about its sign. The presence of a positive exponent in the $\mathrm{CW}$ series would imply that nonlinear instabilities can grow on a $D_{\mathrm{u}}=1 \mathrm{D}$ manifold so putting an intrinsic limitation to the possibility to predict its time evolution over a finite time interval (the so-called horizon of prediction), whatever might be the nature and skill of the algorithm designed for this task. Our confidence in the positiveness of the $\lambda_{1}$ exponents can be reinforced by considering now the $\mathrm{CW}$ series with reversed time (starting from 1997 and ending on 1857). Any direction expanding (contracting) forward in time will contract (expand) backward so that the corresponding exponent will change sign (note that sign corrections are applied in the discussion below and Table II for comparisons). With time reversed, we find again the principal exponents to be positive but smaller than before $\left(\lambda_{1}^{-}(X)=0.032\right.$ and $\lambda_{1}^{-}(Y)=0.018$, the minus superscript indicating time reversal). The exponents associated with the neutral manifolds are $\lambda_{2}^{-}(X)=-0.004$ and $\lambda_{2}^{-}(Y)=-0.001$ and the sums of the exponents always negative as it should be. Moreover these characterizations of the $X$ and $Y$ components of the $\mathrm{CW}$ processed independently from each other are coherent.

All these results strongly suggest that the $\mathrm{CW}$ is an unstable (or equivalently "chaotic") low dimensional deterministic process. From our past experience, we know that the nonlinear analysis of a finite time series dominated by a stochastic component sometimes converges to a finite embedding $D_{E^{*}}$ and (much less convincingly) local $D_{\mathrm{L}}$ dimensions. But until now we found not a single example of such a series providing forward and backward negative exponent sums aud sign-invariant principal exponent.
On the basis of the average principal exponents $\left(\lambda_{1}=\left[\lambda_{1}^{+}+\lambda_{1}^{-}\right] / 2\right)$, we estimate the horizon of prediction $H_{\mathrm{P}}$ for both series by:

$$
H_{\mathrm{P}}=C \cdot \ln (50) / \lambda_{1} .
$$

This somewhat arbitrary definition corresponds to the time necessary for a $1 \%$ RMS error on the system state to grow up to $50 \%$ RMS (for the CW a $\sim 1$ mas error will grow to about 50 mas) along the unstable manifold. The constant $C$ in (8) is required to scale the horizon in days. We obtain 368 days and 276 days for the $X$ and $Y$ wobble components respectively. If these values are to be confirmed, this would mean that reliable predictions of the $\mathrm{CW}$ cannot be given beyond about one year. The observed departure between the horizons for $X$ and $Y$ probably reflects uncertainties in the estimates of the principal Lyapunov exponents. It might also result from some noise or physical process being predominantly "polarized" on the $Y$ component of the wobble. Such a possibility is not unrealistic as for example we noticed before that the trend component of PM is preferentially polarized on that component (see Section 2).

Another fundamental invariant of the $\mathrm{CW}$ dynamics, the Lyapunov dimension $D_{\text {Lyap }}$ is extracted from the data series. It provides a characterization about the way the orbit (as drawn in Fig. 4) fulfills the phase space. It is conjectured that $D_{\text {Lyap }}$ equals the attractor dimension $D_{\mathrm{A}}$. However we found its estimation to be much more robust than the one of $D_{\mathrm{A}}$ based on the correlation integral (Frede and Mazzega, 1999b). The Lyapunov dimension is define from the exponent spectrum by (Kaplan and Yorke, 1979):

$$
D_{\text {Lyap }}=K+\frac{\sum_{j=1}^{K} \lambda_{j}}{\left|\lambda_{K+1}\right|}
$$

with $\sum_{j=1}^{K} \lambda_{j}>0$ and $\sum_{j=1}^{K+1} \lambda_{j}<0$. A striking result is that the estimates of $D_{\text {Lyap }}$ is very robust when considering the forward and backward time series though the Lyapunov spectra exhibit serious (but not sign affecting) departures as reported above. Indeed we find $D_{\text {Lyap }}^{+}(X)=2.34$ to be compared to 
$D_{\text {Lyap }}^{-}(X)=2.52$ and, for the $Y$ series, $D_{\text {Lyap }}^{+}(Y)=$ 2.43 to be compared to $D_{\text {Lyap }}^{-}(Y)=2.41$. The average dimensions of the two series are even in better agreement with $D_{\text {Lyap }}(X)=2.43$ and $D_{\text {Lyap }}(Y)=2.42$ (these are the values quoted in Table II). It should be noted that these fractal values do not characterize the orbits themselves (which are of course differentiable) but the way the bounded domain of phase space is fulfilled by the system state whirling on the attractor. Such noninteger dimension is often associated with chaotic time series (strange attractor).

\section{DISCUSSION}

At this point of our study what have we learned? Probably the most promising break-through is about the usefulness of considering the $\mathrm{CW}$ as a deterministically driven process of low dimension. The assumption of a stochastic origin has always been at the fundament of the various analyses of wobble observations. Such a partiality is well explained from an historical perspective: first, it is only recently that a set of nonlinear concepts and analysis tools are available for an alternative approach; second, the complex behaviours of the forcing geophysical fluids were themselves traditionally recasted in the paradigm of stochastic processes.

The nonlinear data analysis by phase space reconstruction also provides us with qualitative and quantitative results. As illustrations of the first one, we have shown the $\mathrm{CW}$ to evolve in time with a 1D unstable manifold embedded in its dynamics and that any model of the wobble given in form of a system of coupled ordinary differential equations requires three degrees of freedom $\left(D_{\mathrm{L}}=3\right)$. A quantitative bound of about 1 year is found beyond which any attempt to reliably predict the $\mathrm{CW}$ is doomed to failure.

Have we identified the forcing mechanism of the CW? Not yet but this could be hardly done from the analysis of PM observations only. However the various invariants we have extracted from the wobble data series are related to those of the sought forcing. Indeed the dynamics of a linear dissipative pendulum (modellized by Liouville equations, see Lambeck, 1980) does not exhibit any chaotic time evolution. Starting from a non-equilibrium state, the orbit is spiraling during the transcient response, down to the fixed point equilibrium. The corresponding local and Lyapunov dimensions are $D_{\mathrm{L}}^{\text {trans }}=2$ and $D_{\text {Lyap }}^{\text {trans }}=0$ (no unstable manifold) for the transient and $D_{\mathrm{L}}^{\mathrm{fp}}=0$ and $D_{\text {Lyap }}^{\mathrm{fp}}=0$ for the fixed point. All departures from these values observed in the $\mathrm{CW}$ are inherited from the time evolution of the forcing(s).

A similar comment might apply as well to the quality factor $Q$. Is the $Q$ factor estimated from Earth Orientation Parameters relevant to the way the solid Earth relaxes external energy inputs or is it a measure of the dissipative processes at work in the forcing dynamics? We have no direct answer to this question. However the rate of volume shrinking in phase space to a lower dimensional structure (the attractor set) as estimated from the sum of the Lyapunov exponents should give some clue. In particular the relashionship between the working definition of the quality factor $Q$ and the exponent sum should be investigated.

A further step can be done with the nonlinear data analysis. Usually the identification of a forcing field of Earth rotation is done by comparing the corresponding time series (projected in terms of angular momentum or inertia tensor changes) with the measured variation of the relevant rotation parameter. A complementary approach would consist in comparing the variation in stability of both series. In the previous section we derived the global Lyapunov exponents from the Osedelec matrix $S\left(y_{1}, K\right)$ built with all the jacobians of the time series (see Eq. (6)). In the same way, local exponents can be computed from local Osedelec matrices starting at sample $y_{i}$ with $k$ jacobians $(k$ integer). The exponents so obtained give a measure of the average stability (or, more precisely, unstability if we consider a positive exponent) over the $k$ following time steps in the data series. Time variations of the stability with state evolution is a well 

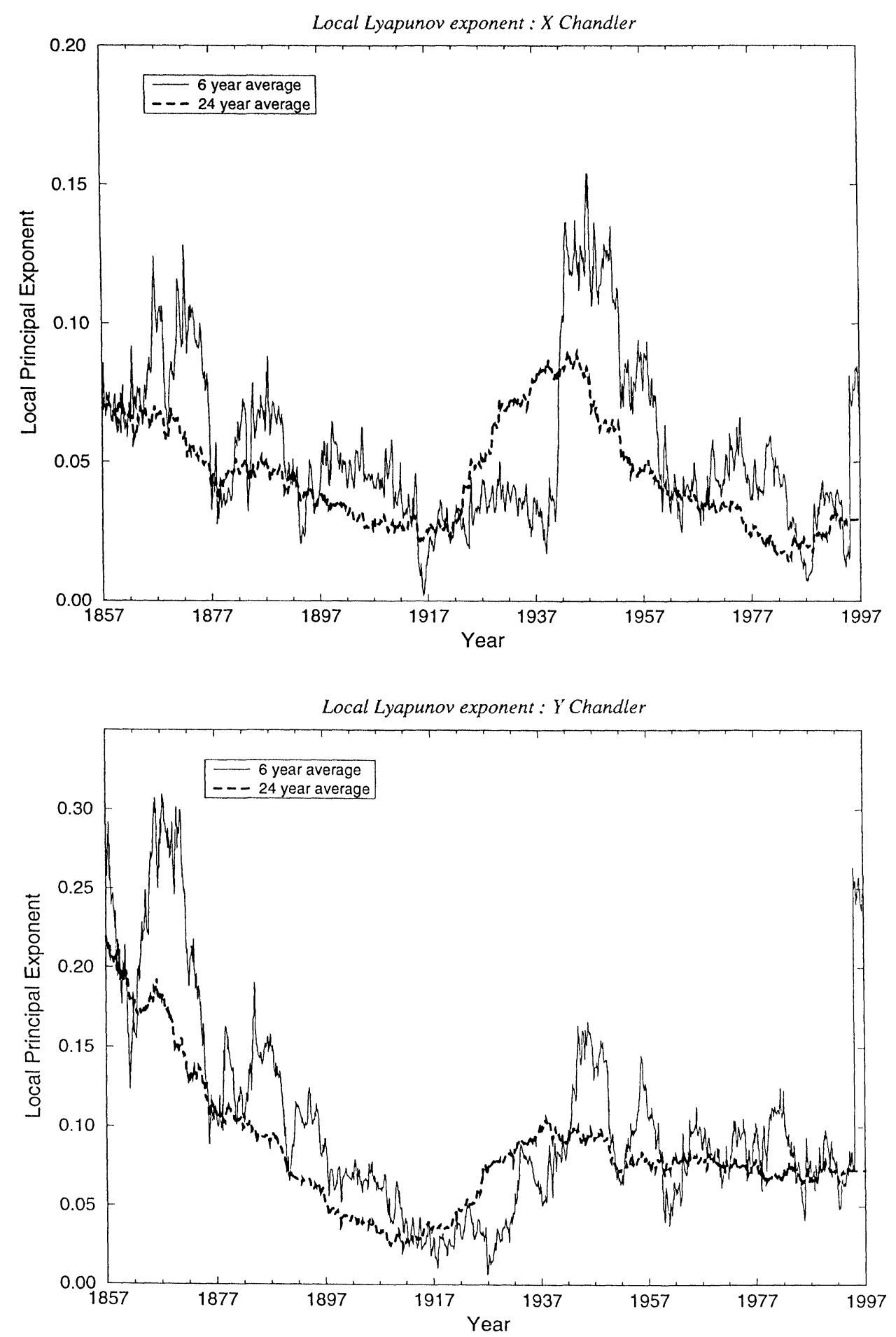

FIGURE 6 Time series of the local principal Lyapunov exponent for the $X$ (upper panel) and $Y$ (lower panel) components of the CW. The plotted exponents are averaged over running windows of 6 and 24 years. High picks in the series correspond to relative losses of stability and reduced horizons of prediction (note that the global exponents are 0.046 and 0.086 for the $X$ and $Y$ series respectively). 
known feature of dynamical systems (Abarbanel et al., 1991).

In Fig. 6 are plotted the time series of the local principal exponents for the $X$ and $Y$ components of the $\mathrm{CW}$. In each case, we computed the average exponents over 6 year and 24 year intervals. The choice for a 6 year window is related to the choice of the same moving window used to separate the annual and CW (Section 2). Siginficant time variations in stability can probably not be retrieved with a higher resolution. The 24 year window is within the accepted range of dissipation rate (deduced from the quality factor; see Section 1) at the $\mathrm{CW}$ period. When increasing the window length, the variations in the local exponent are progressively smoothed out. We notice first that the principal Lyapunov exponents are always positive and exhibit large variations, up to a multiplicative factor $\sim 3$ when compared to the global exponents of Table II. Then the corresponding horizons of prediction are divided by the same factor (see Eq. (8)). For example, in the decades 1860-1870 and 1940-1950, predictions of the future state of the $\mathrm{CW}$ attempted from past observations were intrinsically limited to 4 or 6 months rather than 1 year as stated in the previous Section. Stability variations are even more sensible in the $Y$ component of the wobble. The 1920-1930 decade of weak wobble (see Fig. 1 for $Y$ ) is also quite stable (small exponents) though still chaotic (positive exponents).

Once again we stress on the fact that the observed stability variations are inherited from the unknown forcing of the $\mathrm{CW}$. The time series of a candidate forcing should not only match as much as possible the observed wobble but it should also present the same time evolution of the local Lyapunov exponent. Such a comparison will bring a strong constraint for the identification of the Chandler forcing(s). Such analyses revealed quite successful in recognizing the ocean-atmosphere system coupled in strong El Niño events as a major source of instability in the rapid fluctuations of the Earth rotation (Frede and Mazzega, 1999b).

The various results presented in this study are derived from the data series of the International
Earth Rotation Service (IERS, Paris). Similar computations should be performed with PM series estimated by other institutes and the results compared. These "challenging" series cannot be statistically independent because only a few historical records going back to the middle of the previous century exist. However the data selection, preprocessing and combination can be performed in different ways and lead to more or less diverging results. The global characteristics (time delay, embedding and local dimensions, positivity of the principal Lyapunov exponent) extracted from the IERS data will probably not be affected by such a change in the reference series. But the local characteristics, in particular the local exponents, are likely to change significantly. This kind of result robustness should be checked before proposing detailed interpretations of the time variations of the CW stability.

\section{CONCLUSION}

We give strong evidences that the $\mathrm{CW}$ is driven by a deterministic low dimensional process. The $X$ and $Y$ components of the wobble extracted from PM data series, are embedded in multi-dimensional pseudo-phase spaces with a system of delayed coordinates. The optimal time delay as deduced from the average mutual information function is 105 and 115 days respectively. The embedding dimension is $D_{E^{*}}=4$. Both series can be locally approximated by a three degree of freedom system (local dimension $D_{\mathrm{L}}=3$ ). Then the $\mathrm{CW}$ is shown to be a chaotic process with a $D_{\mathrm{u}}=1 \mathrm{D}$ unstable manifold. The Lyapunov dimension is probably in the range $2.40-2.50$. The nonlinear analysis of both components of the wobble converges to the same kind of conclusions, even when considering the data series with reversed time. The average horizon of prediction is about 1 year. Nevertheless strong fluctuations in the wobble stability can be seen from the time series of the local Lyapunov exponents. These stability crises are inherited from the unknown wobble excitation source and might contribute to its identification. 


\section{Acknowledgements}

This study was supported by the Centre National de la Recherche Scientifique under contract 2E0025 (programme Modèlisation et Simulation Numérique).

\section{References}

Abarbanel, H.D.I., 1996, Analysis of Observed Chaotic Data, Springer Verlag, Berlin, $272 \mathrm{pp}$.

Abarbanel, H.D.I., Brown, R. and Kennel, M.B., 1991, Variation of Lyapunov exponents on a strange attractor, J. Nonlinear Sci., 1, 175-199.

Abarbanel, H.D.I. and Kennel, M.B., 1993, Local false nearest neighbors and dynamical dimensions from observed chaotic data, Phys. Rev. E, 47, 3057-3065.

Carter, W.E., 1981, Frequency modulation of the Chandlerian component of polar motion, J. Geophys. Res., 86(B3), 16531658.

Chao, B.F., 1984, The excitation of the Chandler wobble by the Southern Oscillation, based on ILS data, EOS Trans. Am. Geophys. Un., 65, 859.

Chao, B.F., 1993, Excitation of Earth's polar motion by atmospheric angular momentum variations, 1980-1990, Geophys. Res. Lett., 20(2), 253-256.

Chao, B.F. and Gross, S.R., 1987, Changes in the Earth's rotation and low-degree gravitational field induced by earthquakes, Geophys. J.R. Astr. Soc., 91, 569-596.

Colombo, G. and Shapiro, I.I. 1968, Theorical model for the Chandler wobble, Nature, 217 156-157.

Dehant, V., Wilson, C.R., Salstein, D.A., Chao, B.F., Gross, R.S., Le Provost, C. and Ponte, R.M., 1997, Study of Earth's rotation and geophysical fluids progresses, Eos Trans. $A G U, \mathbf{7 8}(34), 357-360$.

Eckmann, J.P. and Ruelle, D., 1985, Ergodic theory of chaos and strange attractors, Rev. Mod. Physics, 57, 617-656.

Eubanks, T.M., 1993, Variations in the Orientation of the Earth, in Contributions of Space Geodesy to Geodynamics: Earth Dynamics, Smith D.E. and D.L. Turcotte, Eds., Geodynamics Series, Vol. 24, AGU Washington, pp. 1-54.

Fraser, A.M. and Swinney, H.L., 1986, Independent coordinates for strange attractors from mutual information, Phys. Rev. A, 33(2), 1134-1140

Frede, V. and Mazzega, P., 1999a, Detectability of deterministic nonlinear processes in Earth rotation time series: I Embedding, Geophys. J. Int., 137, 551-564.

Frede, V. and Mazzega, P., 1999b, Detectability of deterministic nonlinear processes in Earth rotation time series: II Dynamics, Geophys. J. Int., 137, 565-579.

Furuya, M. and Chao, B.F., 1996, Estimation of period and Q of the Chandler wobble, Geophys. J. Int., 127, 693-702.

Furuya, M., Hamano, Y. and Naito, I., 1996, Quasi-periodic wind signal as a possible excitation of Chandler wobble, $J$. Geophys. Res., 101(B11), 25,537-25,546.

Gray, A. and Thomson, P., 1997, Design of moving-average trend filters using fidelity, smothness and minimum revisions criteria, Bureau of the Census, Stat. Res. Div. Report Series No. RR96/01, 99 pp.

Harrison, D.E. and Larkin, N.K, 1998 ElNiño-Southern Oscillation sea surface temperature and wind anomalies, 1946-1993, Rev. of Geophysics, 36(3), 353-399.

Hide, R. and Dickey, J.O., 1991, Earth's variable rotation, Science, 253, 629-637.

International Earth Rotation Service, 1991, IERS 1990 Annual Report, Central Bureau of IERS, Observatoire de Paris, Paris.

International Earth Rotation Service, 1997, IERS 1996 Annual Report, Central Bureau of IERS, Observatoire de Paris, Paris.

Jeffreys, H., 1940, The variation of latitude, Monthly Not. Roy. Astr. Soc. London, 100(3), 139-155.

Kaplan, J.L. and Yorke, J.A., 1979, Chaotic behaviour in multidimensional difference equations, in Peitgen, H.O. and H.O Walther, Eds., Functional Differential Equations and Approximation of Fixed Points, Springer, Berlin, pp. 204-227.

Lambeck, K., 1980, The Earth's Variable Rotation: Geophysical Causes and Consequences, Cambridge Univ. Press, Cambridge, $449 \mathrm{pp}$.

Le Mouel, J.L., Gire, C. and Hinderer, J., 1985, The flow of the core fluid at the core mantle boundary as a possible mechanism to excite Chandler Wobble, C. R. Acad. Sci. Paris, T. 301(II), 27-32.

McCarthy, D.D. and Luzum, B.J., 1996, Path of the mean rotational pole from 1899 to 1994 Geophys J. Int., 125, 623 629.

Okubo, S., 1982, Is the Chandler period variable? Geophys. J. R. Astr. Soc., 71, 629-646.

Ooe, M., 1978, An optimal complex AR.MA model of the Chandler wobble, Geophys. J.R. Astr. Soc., 53, 445-457.

Sabadini, R., Yuen, D.A. and Boschi, E., 1984, The effects of postseismic motions on the moment of inertia of a stratified viscoelastic Earth with an asthenosphere, Geophys. J. R. Astr. Soc., 79, 727-745.

Shiskin, J., Young, A.H. and Musgrave, J.C., 1967, The X-11 variant of the Census method II seasonal adjustement program, Tech. Paper 15, Bureau of the Census, US Dept. of Commerce, Washington D.C.

Souriau, A., 1986, The influence of earthquakes on the Polar Motion, in Earth Rotation: Solved and Unsolved Problems, Cazenave, A. Ed., Reidel Publ. Comp., pp. 229-240.

Stacey, F.D., 1992, Physics of the Earth, Brookfiled Press, 3rd edition, Brisbane, $513 \mathrm{pp}$

Takens, F., 1981, Detecting strange attractors in turbulence, in Dynamical Systems and Turbulence, Rand, D. and L.S. Young Eds., Lecture Notes in Mathematics, 898, Springer Verlag, Berlin, pp. 366-381.

Vicente, O. and Wilson, C.R., 1997, On the variability of the Chandler frequency, J. Geophys. Res., 102(B9), 20,43920,455 .

Voudràk, J., 1990, Atmospheric and groundwater excitation of polar motion in case of variable Chandler frequency, Bull. Astron. Inst. Czechosl., 41, 211-220.

Wahr, J.M., 1983, The effects of the atmosphere and oceans on the Earth's wobble and on the seasonal variations in the length of day-II Results, Geophys. J. R. Astr. Soc., 74, 451-487. 


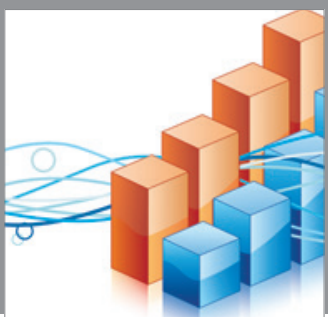

Advances in

Operations Research

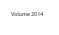

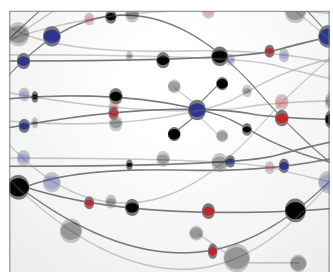

\section{The Scientific} World Journal
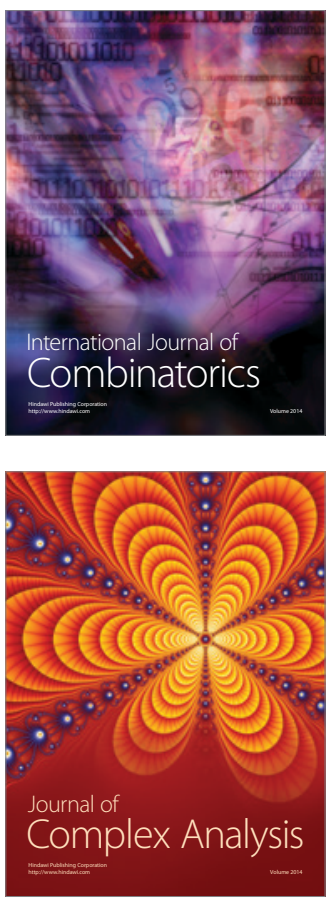

International Journal of

Mathematics and

Mathematical

Sciences
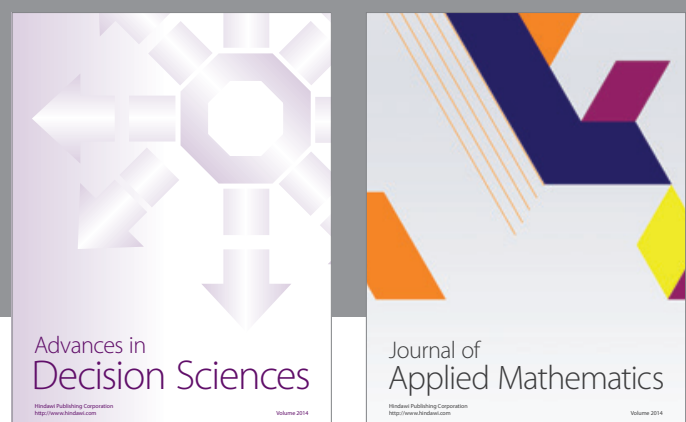

Journal of

Applied Mathematics
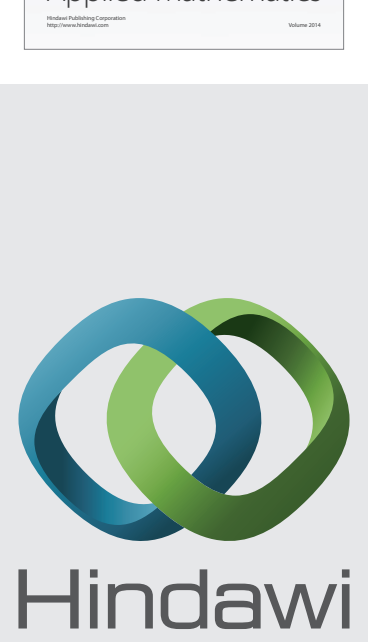

Submit your manuscripts at http://www.hindawi.com
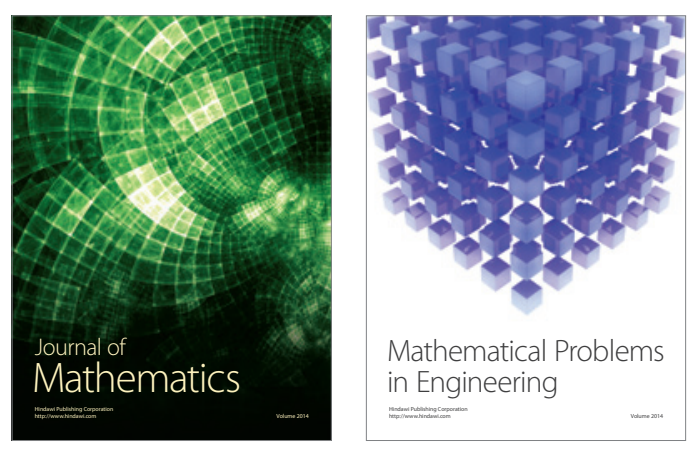

Mathematical Problems in Engineering
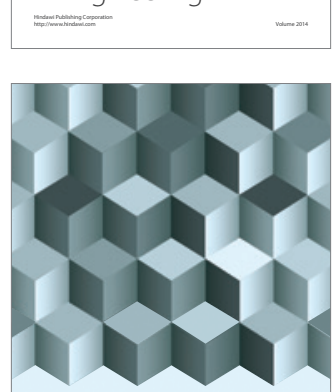

Journal of

Function Spaces
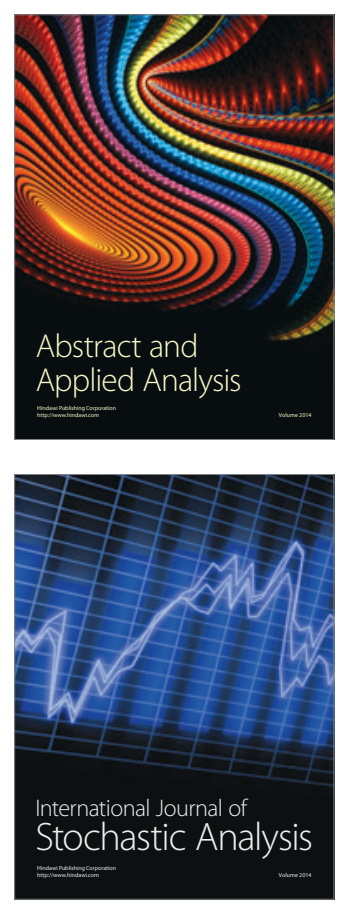

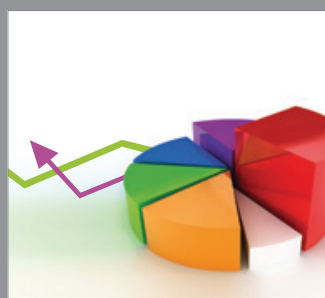

ournal of

Probability and Statistics

Promensencen
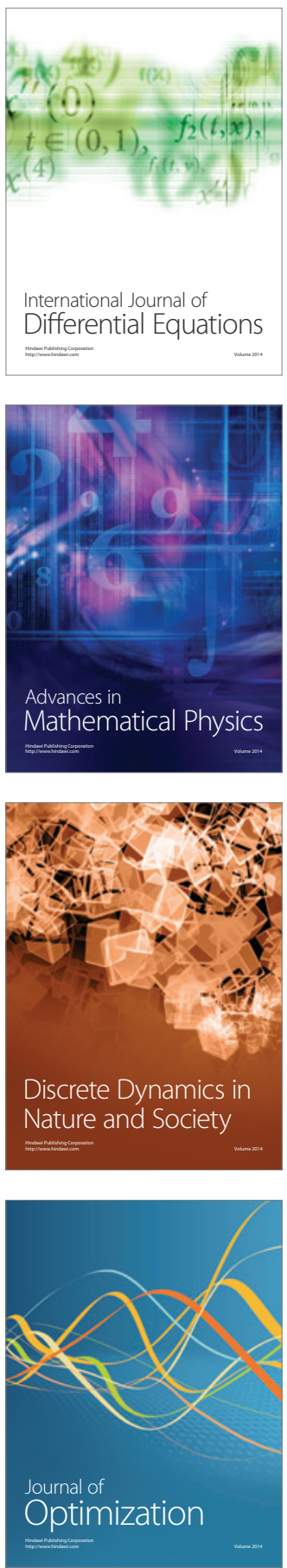